\title{
Probability of earthquake occurrence and magnitude estimation in the post shut-in phase of geothermal projects
}

\author{
Andreas Barth • Friedemann Wenzel . \\ Cornelius Langenbruch
}

Received: 29/05/2011 / Accepted: 16/11/2011 DOI: 10.1007/s10950-011-9260-9

\begin{abstract}
Induced seismicity in geothermal projects is observed to continue after shut-in of the fluid injection. Recent experiments show that the largest events tend to occur after the termination of injection. We use a probabilistic approach based on Omori's law and the Gutenberg-Richter magnitude frequency distribution to demonstrate that the probability of exceeding a certain maximum magnitude still increases after shut-in. This increase is governed by the exponent of Omori's law $q$ and the Gutenberg-Richter $b$-value. For a reduced $b$-value in the post-injection phase the probability of occurrence directly after shut-in can be even higher than the corresponding probability for an on-going injection.

For the reference case of $q=2$ and a $10 \%$ probability at shut-in time $t_{S}$ to exceed a given maximum magnitude we obtain an increase to $14.6 \%$ for $t=2 t_{S}$ at a constant Gutenberg-Richter $b$-value after shut-in. A reduction of the $b$-value by one quarter leads to a probability of $20.5 \%$. If we consider a constant probability level of occurrence for an event larger than a given magnitude at shut-in time, this maximum magnitude increases by 0.12 units for $t=2 t_{S}(0.26$ units for a reduced $b$-value).

For the Soultz-sous-Forêts (France) injection experiment in 2000 recent studies reveal $q=9.5$ and a $b$-value reduction by $14 \%$. A magnitude 2.3 event 9 h after shutin falls in the phase with a probability higher than for the continued injection. The probability of exceeding the magnitude of this post-injection event is determined to $97.1 \%$.
\end{abstract}

\author{
A. Barth · F. Wenzel \\ Karlsruhe Institute of Technology (KIT) \\ Geophysical Inst., Hertzstr. 16, 76187 Karlsruhe, Germany \\ E-mail: a.barth@kit.edu \\ F. Wenzel \\ E-mail: friedemann.wenzel@kit.edu \\ C. Langenbruch \\ Freie Universität Berlin, Inst. of Geological Sciences \\ Malteserstr. 74-100, 12249 Berlin, Germany \\ E-mail: Cornelius@geophysik.fu-berlin.de
}


Keywords induced seismicity · shut-in · post injection · probability · EGS · b-value · Soultz-sous-Forêts · Basel

\section{Introduction}

It is known that fluid injections at geothermal sites, which are performed to develop the reservoirs, can induce low magnitude earthquakes in critically stressed zones of the surrounding rock. Even after shut-in, that is, after the pressurised fluid injection into the borehole is stopped, a significant number of seismic events can occur (Parotidis and Shapiro 2004; Parotidis et al 2004). The understanding, characterisation, and forecasting of post-injection events is particularly important, because during recent geothermal projects such as Soultz-sous-Forêts (France, Charléty et al 2007), Basel (Switzerland, Häring et al 2008), and Landau (Germany) it has been observed that the largest earthquakes tend to occur after shut-in. This makes it still more difficult to control such events. Those earthquakes have had a large impact in society and understanding their temporal occurrence was identified as one major goal of geothermal research (Majer et al 2007). There is speculation that the largest earthquakes are therefore causally related to the shut-in as if the stop of injection would lead to the larger earthquake.

In general, the behaviour of seismicity triggering in space and time is controlled by the relaxation process of stress and pore pressure perturbation that was initially created at the injection source. This relaxation process can be approximated by linear pressure diffusion in the pore fluid of rocks. Following the Mohr-Coulomb failure criterion the resulting increase in pore pressure can lead to rock failure along pre-existing, sub-critically stressed cracks.

Recent findings from Langenbruch and Shapiro (2010) suggest that the temporal development of the seismicity rate is controlled by the stability of the preexisting fracture system. More precisely, if the stress state of the fractures is close to failure, that is, a small increase of pore pressure will be sufficient to bring the fractures to failure, the seismicity rate will be the highest close before and after the shut-in and the decay rate after shut-in will be slow. This may also be the reason for larger magnitude events around the shut-in time. In contrast, a fracture system consisting of fractures characterised by a more stable stress state (a significant increase in pore pressure is needed to bring the fractures to failure) results in a faster decay of seismicity and thus to a lower probability of large events. Wenzel et al (2010) determined theoretical event probabilities and expected magnitudes for post-injection seismicity, assuming a constant $b$-value.

Large post-injection events can be related to a decrease of the GutenbergRichter (G-R) $b$-value, leading to a relatively higher number of large events. Bachmann et al (2011) calculated an average decrease of $26 \%$ of the $b$-value after the shut-in for the Basel geothermal project. At Soultz-sous-Forêts a decrease of $14 \%$ was determined for a stimulation experiment in 2000 (Cuenot et al 2008).

In this paper we demonstrate that a reduced $b$-value after shut-in of injection increases the event probabilities for large events in the context of the "Seismicity Based Reservoir Characterization Theory" (SBRC). Temporary, probabilities can be even higher than for a continued injection. However, on a longer time scale higher probabilities have to be expected for an on-going experiment. Assuming a 
constant probability level, our considerations result in an increase of the largest expected magnitude after shut-in, especially for a reduced $b$-value.

\section{Theory}

In statistical seismology there are two fundamental laws, namely the Omori law, which describes the decay rate of aftershock activity after tectonically driven earthquakes and the Gutenberg-Richter (G-R) relation describing the magnitude frequency distribution of earthquakes. It was observed and verified in recent works that both fundamental laws are also valid in the context of injection-induced seismicity (Shapiro et al 2007; Langenbruch and Shapiro 2010).

We describe the fluid injection by a point source in a permeable fluid-saturated medium with pre-existing fractures and assume that the fluid is liberated from this source with constant strength until the shut-in time $t_{S}$. According to Shapiro et al (2007) this leads to a constant seismicity rate $\bar{\nu}_{0}$ for earthquakes with magnitudes larger than the lower threshold of complete recording $m_{0}$ following the G-R relation:

$$
\log \bar{\nu}_{0}=a-b m_{0} .
$$

For another magnitude $M$ the parameter $a$ may be substituted from eq. 1 :

$$
\log \bar{\nu}_{M}=\log \bar{\nu}_{0}+b m_{0}-b M
$$

resulting in a constant seismicity rate $\bar{\nu}_{M}$ :

$$
\bar{\nu}_{M}=\bar{\nu}_{0} \cdot 10^{-b\left(M-m_{0}\right)} .
$$

Omori's law can be utilised to describe the decay of the seismicity rate $\nu_{M}(t)$ with magnitudes larger than $M$ after shut-in of injection in the following modified form (Langenbruch and Shapiro 2010):

$$
\nu_{M}(t)=\bar{\nu}_{M}\left(\frac{t_{S}}{t}\right)^{q}=\bar{\nu}_{0} \cdot 10^{-b\left(M-m_{0}\right)}\left(\frac{t_{S}}{t}\right)^{q},
$$

with time $t \geq 0$ from injection start and the exponent $q \geq 1$, which controls the decay of seismicity. At shut-in time $\left(t=t_{S}\right)$ the seismicity rate equals the constant seismicity rate $\bar{\nu}_{M}$ during injection. While $q=2$ is often used as a reference value, the analysis of seismicity data from geothermal projects recently suggests even higher values for $q$, if the reactivated fracture system is in a stable state of stress (Langenbruch and Shapiro 2010).

We assume that the induced earthquakes are temporally uncorrelated, i.e. Poissonian distributed (Shapiro et al 2010; Langenbruch et al 2011). If the seismicity rate is constant the Poisson process is called homogeneous. For a constant $b$-value the probability that no earthquake in excess of $M$ occurs between the initiation of injection and some time $t$ is (Wenzel et al 2010)

$$
\begin{aligned}
P_{0}(M, t) & =\exp \left(-\bar{\nu}_{M} t\right) \\
& =\exp \left(-\bar{\nu}_{0} \cdot 10^{-b\left(M-m_{0}\right)} t\right) \\
& =\exp \left(-\bar{\nu}_{0} \cdot e^{-\beta\left(M-m_{0}\right)} t\right),
\end{aligned}
$$


with $\beta=b \cdot \ln 10 \approx 2.3 \cdot b$. The distribution is of Gumbel type, which is not surprising as we look for the extreme value of $\bar{\nu}_{M} \cdot t$ earthquakes.

If the intensity of the Poisson process varies with time (and thus the seismicity rate) it becomes inhomogeneous. The probability that no earthquake in excess of $M$ occurs between the initiation of injection and some time $t$ can be written as

$$
P_{0}(M, t)=\exp \left(-\int_{0}^{t} \nu_{M}(\tau) \mathrm{d} \tau\right)
$$

For the shut-in time $\left(t=t_{S}\right)$ we get for the probability that magnitude $M$ is not exceeded between time 0 and $t_{S}$ :

$$
\ln \frac{1}{P_{0}\left(M, t_{S}\right)}=\bar{\nu}_{M} t_{S}
$$

Using the decaying seismicity rate for the time after shut-in $t \geq t_{S}$ we obtain

$$
\begin{aligned}
\ln \frac{1}{P_{0}(M, t)} & =\bar{\nu}_{M} t_{S}+\int_{t_{S}}^{t} \nu_{M}(\tau) \mathrm{d} \tau \\
& =\bar{\nu}_{M} t_{S} \cdot\left(1+Q_{S}(t)\right),
\end{aligned}
$$

with

$$
Q_{S}(t)=\frac{1-\left(t / t_{S}\right)^{1-q}}{q-1} .
$$

Thus it is clear that the probability $P_{0}$ of not exceeding magnitude $M$ is still decreasing after shut-in. If the injection is not shut off but continues some time beyond $t \geq t_{S}$ we have the trivial relation

$$
\ln \frac{1}{P_{0}(M, t)}=\bar{\nu}_{M} t_{S} \cdot\left(1+\frac{t-t_{S}}{t_{S}}\right)=\bar{\nu}_{M} t .
$$

Hence the probability to exceed magnitude $M$ (that is $1-P_{0}$ ) still increases after the original shut-in time. The relaxation of pore pressure after termination of injection however, makes sure that seismicity stops when the pore-pressures fall below a critical level.

So far we considered the $b$-value as constant over time. However, recent studies indicate a decrease of the $b$-value after shut-in with an increasing number of larger events (Bachmann et al 2011; Cuenot et al 2008). Assuming a changed $b$-value $b^{\prime}=x b$ for a constant rate of the threshold magnitude $m_{0}$, it follows for the postinjection occurrence rate $\nu_{M}^{\prime}$ of events larger than magnitude $M$ :

$$
\begin{aligned}
\nu_{M}^{\prime}(t) & =\bar{\nu}_{0} \cdot 10^{-b^{\prime}\left(M-m_{0}\right)}\left(\frac{t_{S}}{t}\right)^{q} \\
& =\bar{\nu}_{0} \cdot\left(10^{-b\left(M-m_{0}\right)}\right)^{x}\left(\frac{t_{S}}{t}\right)^{q} .
\end{aligned}
$$

Inserting eq. 4 yields

$$
\nu_{M}^{\prime}(t)=\nu_{M}(t) \cdot 10^{-b\left(M-m_{0}\right)(x-1)} .
$$




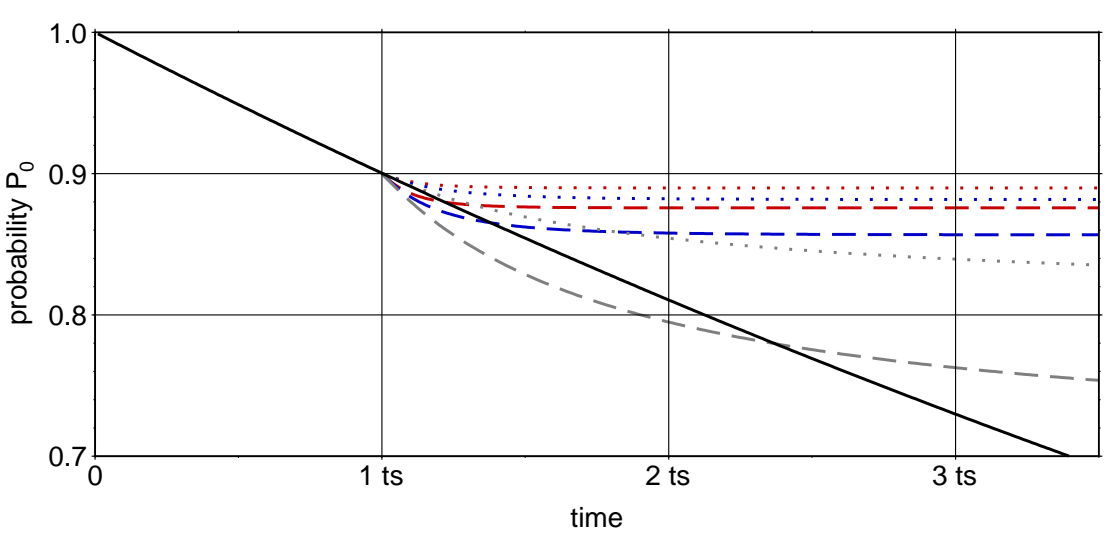

Fig. 1 Probability $P_{0}$ of not exceeding a maximum magnitude $M$ with time (shut-in time $\left.t_{S}\right)$. The solid black line corresponds to continued injection. Other lines show $P_{0}$ after shut-in for different $q$-values: $q=2$ (grey), $q=6$ (blue), and $q=10$ (red). Dotted lines: constant $b$-value $b=1.5$. Dashed lines: $b$-value reduced by $25 \%$, with respect to the injection phase.

Accordingly, the probability of not exceeding magnitude $M$ after shut-in (compare eq. 8) decreases even further:

$$
\begin{aligned}
\ln \frac{1}{P_{0}^{\prime}(M, t)}= & \bar{\nu}_{M} t_{S}+\int_{t_{S}}^{t} \nu_{M}^{\prime}(\tau) \mathrm{d} \tau \\
= & \bar{\nu}_{M} t_{S} \cdot \\
& \left(1+10^{-b\left(M-m_{0}\right)(x-1)} \cdot Q_{S}(t)\right) .
\end{aligned}
$$

Let us give an example (Fig. 1): If the probability to exceed magnitude $M$ at the time of the shut-in is given by $1-P_{0}=10 \%$, this probability increases to $1-P_{0}=14.6 \%$ (dotted grey line) considering all events occurring until $t=2 t_{S}$ and an exponent of $q=2$ (eq. 8). The corresponding values for $q=6$ and $q=10$ are $11.8 \%$ and $11.0 \%$, respectively. For the theoretical limit of $q=1$ (not to be expected in nature) the probability reaches $1-P_{0}=16.3 \%$ (Wenzel et al 2010). Assuming an initial value of $b=1.5$ reduced by $25 \%$ for the post-injection phase and a magnitude of interest one order higher than the magnitude of completeness $\left(M-m_{0}=1\right)$, it follows $1-P_{0}=20.5 \%$ at $t=2 t_{S}$ (eq. 13, dashed grey line in Fig. 1). Even the corresponding value for an on-going injection is lower at that time: $1-P_{0}=18.9 \%$ (eq. 10, black solid line). On a long-term perspective the event probability for a continued experiment indeed is higher than after stopping the injection.

Next we consider the probabilistically determined largest earthquake from a different point of view. Given a constant probability level of occurrence and a constant $b$-value after shut-in, we study the magnitude changes. The probability not to exceed magnitude $M$ until shut-in time $t_{S}$ is (eq. 3,7 )

$$
\ln \frac{1}{P_{0}\left(M, t_{S}\right)}=\bar{\nu}_{M} \cdot t_{S}=\bar{\nu}_{0} \cdot 10^{-b\left(M-m_{0}\right)} \cdot t_{S}
$$


From this $M$ can be calculated if $\bar{\nu}_{0}$ and $b$ are known. Keeping the probability level constant we can ask how would the maximum magnitude increase beyond $M$ if we (a) continue injection until $t \geq t_{S}$ and (b) stop injection and wait until $t \geq t_{S}$.

The general implicit formula for both cases is

$$
P_{0}\left(M, t_{S}\right)=P_{0}(M+\Delta M, t) .
$$

In case (a) we get after some manipulations

$$
\Delta M=\frac{1}{b} \log \left(t / t_{S}\right)
$$

Thus at a constant probability that no earthquake larger than $M$ occurs, the change in magnitude by extending the injection time from $t_{S}$ to $t=2 t_{S}$ is

$$
\Delta M=\frac{1}{b} \log (2) \approx \frac{0.3}{b} \approx 0.2,
$$

assuming a $b$-value of 1.5 . The general shut-in case (b) for a constant $b$-value leads to

$$
\Delta M=\frac{1}{b} \log \left(1+Q_{S}(t)\right) .
$$

For the reference value of $q=2$ and again $t=2 t_{S}$, we find

$$
\Delta M=\frac{\log 1.5}{b} \approx 0.12 .
$$

For the theoretical limit of $q=1$ the magnitude increases further $\left(\Delta M\left(b=1.5, t=2 t_{S}\right) \approx 0.15\right)$ (Wenzel et al 2010).

Now, applying again a changed $b$-value $b^{\prime}=x b$ and setting

$$
P_{0}\left(M, t_{S}\right)=P_{0}^{\prime}(M+\Delta M, t),
$$

it follows from eq. 7 and 13

$$
10^{x b \Delta M}-10^{(x-1) b \Delta M}=Q_{S}(t) \cdot 10^{-b\left(M-m_{0}\right)(x-1)} .
$$

This transcendental equation has no general analytical solution for $\Delta M$. The numerical solution for the exemplary values from before $(b=1.5,1-x=25 \%, q=2$, and $\left.M-m_{0}=1\right)$ results in $\Delta M=0.26$ at the double shut-in time $t=2 t_{S}$.

\section{Soultz-sous-Forêts}

We apply the above theory to real data that was recorded during a hydraulic stimulation experiment at Soultz-sous-Forêts (France) in 2000 (Cuenot et al 2008). The experiment started on June 30, 19h 00 GMT and was stopped after $141 \mathrm{~h}$. Injection rates were increased from $30 \mathrm{l} / \mathrm{s}$ to $50 \mathrm{l} / \mathrm{s}$ in two steps. $102 \mathrm{~h}$ after initiation of the injection a magnitude 2.5 (duration magnitude, Charléty et al 2007) earthquake occurred. During the post-injection time seismicity decreases quantitatively (see Fig. 2), while a magnitude 2.3 event took place $9 \mathrm{~h}$ after shutin. Ten days after the first shut-in and one day after a post-fracturation test 


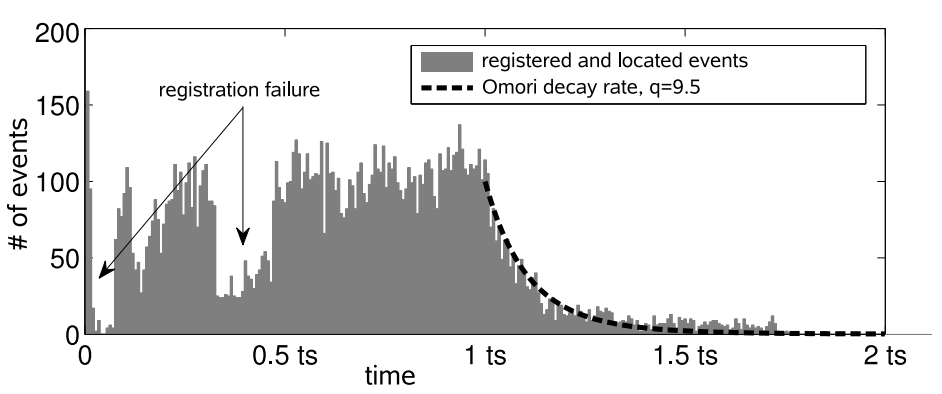

Fig. 2 Temporal development of detected seismicity in the Soultz-sous-Forêts stimulation experiment in 2000. Red bars indicate the number of seismic events per time step. Arrows show gaps in the observation due to a not operating monitoring system. After shut-in the dashed line shows the best fit for Omori's law with $q=9.5$.

with lower injection rates than before $(15-30 \mathrm{l} / \mathrm{s})$ the largest earthquake with a magnitude 2.6 occurred (Cuenot et al 2008; Charléty et al 2007).

Cuenot et al (2008) analysed the time development of magnitude frequencies during the stimulation experiment. They found $b$-value variations within a range from 1.0 to 1.7. After the shut-in the $b$-value decreased from 1.4 to 1.21 (14\% reduction). The portion of microseismic events with magnitudes higher or equal 2 with respect to the total amount of seismicity in the post-injection phase was four times higher than during injection. However, before stopping injection a large deficit in higher magnitude events $M \geq 1.8$ was observed. To calculate the probabilities of post shut-in seismicity according to our theory we determine the $b$-value during the stimulation period for magnitudes larger than $m_{0}=1.8$. To analyse the G$\mathrm{R}$ magnitude-frequency distribution we apply the maximum-likelihood estimation after Aki (1965) and Utsu (1965):

$$
b=\left(\bar{m}-m_{0}\right)^{-1} \cdot \log (e),
$$

with the mean magnitude $\bar{m}$ and a magnitude level of data completeness $m_{0}$. Under the assumption that the datasets are samples from a population obeying the G-R relation, this formulation is equivalent to the classical G- $\mathrm{R}$ distribution. For the dataset of $N=69$ events with magnitudes $M \geq 1.8$ of the stimulation experiment at Soultz-sous-Forêts the cumulative number $N$ of earthquakes with magnitudes greater or equal $M$ is

$$
\log (N)=a-b M=6.15-2.45 \cdot M .
$$

After Aki (1965) the standard deviation of the $b$-value is approximately

$$
\sigma(b)=b / \sqrt{N}=0.29 .
$$

The decay of seismicity for the post-injection phase can be well approximated by the modified Omori law. For the calculation of $q$ we use the catalogue of recorded events shown in Fig. 2. A value of $q=9.5$ results in the best fit to the observed post-injection seismicity (compare Langenbruch and Shapiro 2010).

We use both the determined $q$ - and $b$-value to calculate the probability of not exceeding magnitude $M$ during injection (eq. 5) and after shut-in for a constant $b$-value (eq. 8 ) and a reduced one (eq. 13). Figure 3 shows the probabilities for 


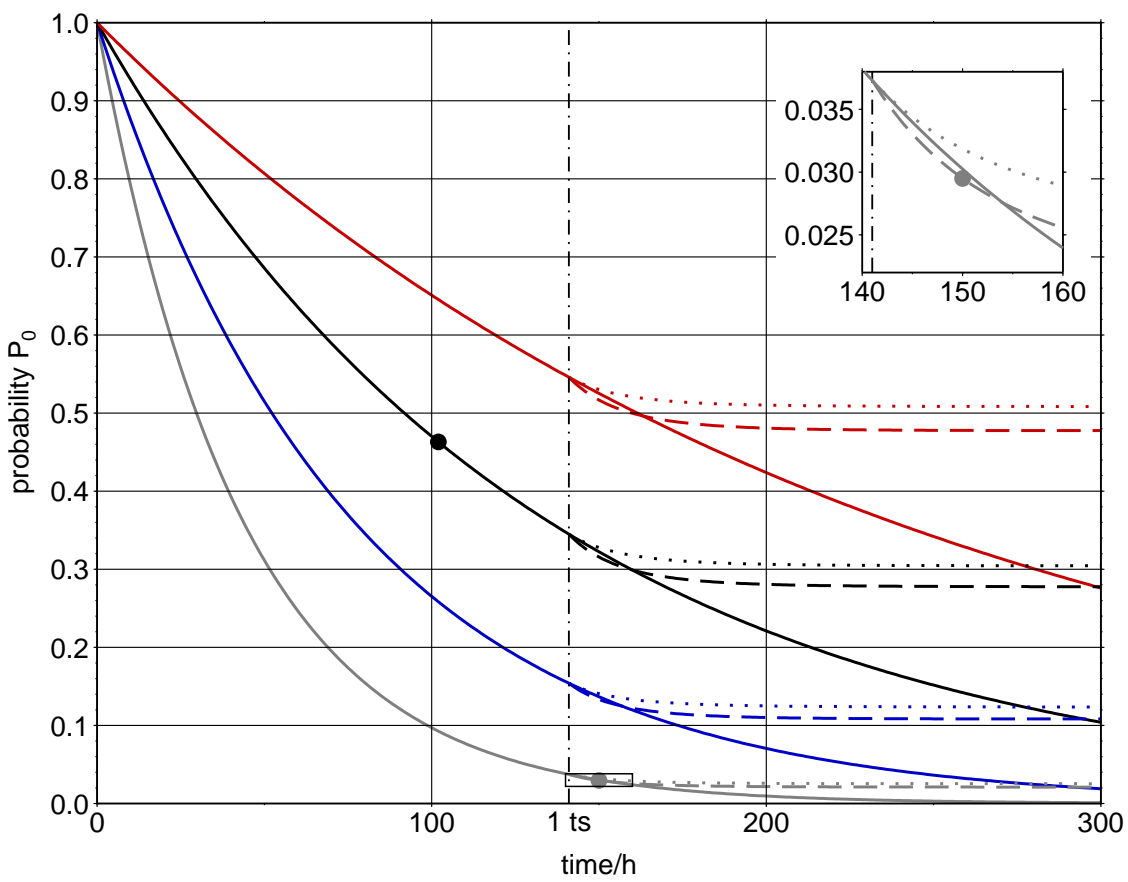

Fig. 3 Probability $P_{0}$ of not exceeding a maximum magnitude $M$ with time for the Soultzsous-Forêts experiment 2000 (shut-in time $t_{S}=141 \mathrm{~h}$ ). Colours indicate different maximum magnitudes $M$ : Grey: $M=2.3$, blue: $M=2.4$, black: $M=2.5$, and red: $M=2.6$. Solid lines correspond to continued injection. Dotted lines show $P_{0}$ after shut-in for a constant $b$ value $b=2.45$, dashed lines for a $b$-value reduced by $14 \%$ (see text). The two dots mark the probabilities for the occurrence of the two largest real events $M=2.5$ (black) and $M=2.3$ (grey). The latter occurred $9 \mathrm{~h}$ after shut-in (see enlarged inset).

magnitudes 2.3 to 2.6. The lower the maximum magnitude, the lower are the probabilities $P_{0}$ of not exceeding a certain magnitude. Thus the probability of exceeding the largest earthquake during injection $(M=2.5)$ was $1-P_{0}=53.7 \%$ until the occurrence time of this event. In addition to the general decrease of $P_{0}$ with time, a reduced $b$-value after shut-in reduces this probability even more. For a certain time $P_{0}$ even falls beneath the probability for a continued injection (see Fig. 1, 3). The post-injection $M=2.3$ event occurred in that phase $(9 \mathrm{~h}$ after stopping the injection) and had a probability of $1-P_{0}=97.1 \%$ to occur.

\section{Conclusion}

We have shown that based on a modified Omori law and a Gutenberg-Richter distribution the probability $P_{0}$ of not exceeding a maximum magnitude during injection and after its termination can be determined. After termination of injection $P_{0}$ strongly depends on the Gutenberg-Richter $b$-value and the exponent $q$ of the modified Omori law. Two characteristic values have been calculated for the postinjection phase and the case of an on-going injection: (a) the continuing decrease of $P_{0}$ and (b) the increase of the maximum magnitude given a constant probabil- 
ity level of occurrence. For (a) we find an increase of $1-P_{0}$, i.e. the probability of exceeding a maximum magnitude, from a given value of $10 \%$ at shut-in time $t_{S}$ to $14.6 \%$ for time $t=2 t_{S}(q=2)$. A $b$-value reduced by $25 \%$ after shut-in results in an even higher value of $1-P_{0}=20.5 \%$. At the same time a continued injection corresponds to $1-P_{0}=18.9 \%$. The maximum magnitude (b) increases for $t=2 t_{S}$ by 0.2 for a continued injection and for the shut-in case by 0.12 (constant $b$-value) and 0.26 (reduced $b$-value), respectively.

The application of the theory to the stimulation test at Soultz-sous-Forêts in the year 2000 reveals a $97.1 \%$ probability to exceed the magnitude $M=2.3$ earthquake that happened $9 \mathrm{~h}$ after shut-in. The $b$-value of the magnitude frequency distribution was reduced by $14 \%$ with respect to the injection phase. The seismic event occurred during the time when the probability was higher than it would have been for an on-going injection.

While high $q$-values indicate only a small increase of the probability to exceed the maximum magnitude, lower $q$-values result in a higher probability increase after shut-in. Additionally, a reduced $b$-value in the post-injection phase can be associated with the occurrence of large magnitude earthquakes after shut-in and thus with an increase of seismic risk.

Acknowledgements We thank an anonymous reviewer for helpful and constructive remarks. Figures were made using GMT (Wessel and Smith 1998).

\section{References}

Aki K (1965) Maximum likelihood estimate of $\mathrm{b}$ in the formula $\log \mathrm{n}=\mathrm{a}-\mathrm{bm}$ and its confidence limits. Bull Earthq Res Inst 43:237-239

Bachmann CE, Wiemer S, Woessner J, Hainzl S (2011) Statistical analysis of the induced Basel 2006 earthquake sequence: introducing a probability-based monitoring approach for enhanced geothermal systems. Geophysical Journal International 186(2):793-807, DOI 10.1111/j.1365-246X.2011.05068.x

Charléty J, Cuenot N, Dorbath L, Dorbath C, Haessler H, Frogneux M (2007) Large earthquakes during hydraulic stimulations at the geothermal site of Soultz-sousForêts. International Journal of Rock Mechanics and Mining Sciences 44(8):10911105, URL http://www.sciencedirect.com/science/article/B6V4W-4P83DCB1/2/e4abd1c6beff9f97e6a69290d098642d

Cuenot N, Dorbath C, Dorbath L (2008) Analysis of the microseismicity induced by fluid injections at the EGS site of Soultz-sous-Forêts (Alsace, France): Implications for the characterization of the geothermal reservoir properties. Pure Appl Geophys 165(5):797828, DOI 10.1007/s00024-008-0335-7

Häring MO, Schanz U, Ladner F, Dyer BC (2008) Characterisation of the Basel 1 enhanced geothermal system. Geothermics 37(5):469-495

Langenbruch C, Shapiro SA (2010) Decay rate of fluid induced seismicity after termination of reservoir stimulations. Geophysics 75(6):Ma53-Ma62, DOI 10.1190/1.350600

Langenbruch C, Dinske C, Shapiro SA (2011) Inter event times of fluid induced earthquakes suggest their poisson nature. Geophys Res Lett 38:L21,302, DOI 10.1029/2011GL049474

Majer EL, Baria R, Stark M, Oates S, Bommer J, Smith B, Asanuma H (2007) Induced seismicity associated with enhanced geothermal systems. Geothermics 36(3):185-222, URL http://www.sciencedirect.com/science/article/B6VCN-4NMTYWT1/2/762198be91b7010a814835758af06a85

Parotidis M, Shapiro SA (2004) A statistical model for the seismicity rate of fluid-injectioninduced earthquakes. Geophys Res Lett 31(17):L17,609, DOI 10.1029/2004GL020421

Parotidis M, Shapiro SA, Rothert E (2004) Back front of seismicity induced after termination of borehole fluid injection. Geophys Res Lett 31(2):L02,612, DOI 10.1029/2003GL018987 
Shapiro SA, Dinske C, Kummerow J (2007) Probability of a given-magnitude earthquake induced by a fluid injection. Geophys Res Lett 34:L22,314, DOI 10.1029/2007GL031615.

Shapiro SA, Dinske C, Langenbruch C, Wenzel F (2010) Seismogenic index and magnitude probability of earthquakes induced during reservoir fluid stimulations. The Leading Edge 29(3):304-309, DOI 10.1190/1.3353727, URL http://tle.geoscienceworld.org/cgi/content/abstract/29/3/304

Utsu $\mathrm{T}$ (1965) A method for determining the value of $\mathrm{b}$ in a formula $\log \mathrm{n}=\mathrm{a}-\mathrm{bm}$ showing the magnitude-frequency relations for earthquakes. Geophys Bull Hokkaido Univ 13:99-103

Wenzel F, Barth A, Langenbruch C, Shapiro S (2010) Occurrence probability and earthquake size of post shut-in events in geothermal projects. In: Ritter J, Oth A (eds) Proceedings of the Workshop Induced Seismicity", ECGS Blue Book, 30, Luxembourg City, 15-17 November 2010

Wessel P, Smith WHF (1998) New, improved version of Generic Mapping Tools released. EOS, Trans AGU 79:579pp 